\title{
Stability analysis for coupled systems with time-varying coupling structure
}

Jiqiang Feng ${ }^{1 *}$ and Xiaojia Luan ${ }^{2}$

\section{${ }^{*}$ Correspondence:}

fengjqszu@163.com

${ }^{1}$ College of Mathematics and

Statistics, Shenzhen University,

Shenzhen, 518060, P.R. China

Full list of author information is

available at the end of the article

\section{Introduction}

During the past few decades, coupled systems (CSs) have been used to model a wide variety of systems in many fields, such as physics [1-3], biology [4-6] and social science [7]. The general CSs can be described as follows:

$$
\frac{\mathrm{d} x_{k}(t)}{\mathrm{d} t}=f_{k}\left(x_{k}(t), t\right)+\sum_{h=1}^{l} a_{k h} H_{k h}\left(x_{k}(t), x_{h}(t)\right), \quad k=1,2, \ldots, l, t \geq 0,
$$

where $f_{k}$ and $H_{k h}$ are continuous functions, and $a_{k h}$ represents the coupling strength. At the same time, it is of great importance to analyze the stability of CSs, because most applications of CSs heavily depend upon their stability. There are lots of results on CSs with time-invariant coupling structure having been reported (see [1, 4, 5, 8-10] and the references therein). In [11], the stability problem for a multi-group SIRS epidemic model was investigated. Guo et al. [12] considered the stability of a stochastic neural networks with infinite delay and Markovian switching. In [13, 14], Li et al. used graph theory to explore the global stability for general coupled systems of ordinary differential equations. Following this pioneering work in $[13,14]$, many scholars have studied the dynamics of CSs by this technique and obtained a number of conclusions [15-23]. Moreover, the method has been extended to stochastic systems [24-28], discrete time systems [29], and time delay systems [30].

(c) The Author(s) 2017. This article is distributed under the terms of the Creative Commons Attribution 4.0 International License (http://creativecommons.org/licenses/by/4.0/), which permits unrestricted use, distribution, and reproduction in any medium, provided you give appropriate credit to the original author(s) and the source, provide a link to the Creative Commons license, and indicate if changes were made. 
Generally speaking, the coupling structure of CSs is not constant. For example, in biomathematics, the dispersal rate of some species among different groups changes over time. When a natural disaster occurs, such as earthquakes and flood, some species will migrate to a safe place, then the dispersal rate of them will improve significantly. In epidemiology, the transmission rate of infectious diseases is also time-varying. For instance, population migration across regions increases greatly during the high season, and then the transmission rate of infectious diseases will be higher. However, when some regions discontinue with each other due to some reasons, the transmission rate of infectious diseases will be lower. All these facts can illustrate that time-varying coupling structure should not be neglected. Thus, we should take this time-varying coupled behavior into account, which can help us investigate the persistence of species and control infectious diseases. Consequently, it is essential for us to investigate the coupled systems with time-varying coupling structure (CSTCS). Unfortunately, as far as we know, few papers mentioned in the existing literature consider CSTCS.

Until now, many researchers, including us, have given some results of global stability of CSs. However, in our previous work we do not consider the time-varying coupling structure. In this paper, in order to make up for the defect of our previous work and characterize CSs reasonably, we will devote ourselves to the investigation of CSTCS. Generally, compared with system (1), $a_{k h}(t) H_{k h}\left(x_{k}(t), x_{h}(t)\right)$ may more reasonably be used to describe the interactions within a group or among different groups in the course of the dispersal. However, it is intricate to study the dynamics of CSTCS, since constructing a global Lyapunov function and estimating the symbol of its derivative are complex and technical under the circumstances of the time-varying coupling structure.

Motivated by the above discussions, in this paper, by introducing the time-varying coupling structure, we present a class of novel CSs that is CSTCS. Based on the graph theory and the Lyapunov method, a systematic method is established to construct a global Lyapunov function for CSTCS. Moreover, sufficient criteria ensuring the stability of CSTCS can be obtained. Furthermore, we consider a predator-prey model with time-varying dispersal. Meanwhile, stability criteria for it are presented, respectively. Then a numerical example is given to illustrate the effectiveness of our results.

The paper is outlined as follows. In Section 2, we introduce several preliminaries and give the formulation of the CSTCS. In Section 3, sufficient criteria of the stability for CSTCS are obtained. The stability results for predator-prey model with time-varying dispersal in Section 4. Finally, a numerical example is given in Section 5.

\section{Preliminaries and model formulation}

\subsection{Mathematical preliminaries}

For the sake of simplicity, the following notations are used in this paper. Let $\mathbb{Z}^{+}=\{1,2, \ldots\}$, $\mathbb{L}=\{1,2, \ldots, l\}, \mathbb{R}_{+}^{1}=[0,+\infty), n=\sum_{k=1}^{l} n_{k}$ for $n_{k} \in \mathbb{Z}^{+}$, and $\mathbb{R}^{n}$ denote $n$-dimensional Euclidean space. And we write $C^{1,1}\left(\mathbb{R}^{n} \times \mathbb{R}_{+}^{1} ; \mathbb{R}_{+}^{1}\right)$ for the family of all nonnegative functions $V(x, t)$ on $\mathbb{R}^{n} \times \mathbb{R}_{+}^{1}$ that are continuously once differentiable at $x$ and $t$.

In this paper, use $(\mathcal{G}, A)$ to represent the digraph with $l$ vertices. Let $V(\mathcal{G})$ denote the set of vertices in $\mathcal{G}$; without loss of generality, let $V(\mathcal{G})=\mathbb{L}$.

In what follows, we show an important lemma in [14] which will be used in the proof of our main results. 
Lemma 1 Assume $n \geq 2$. Let $c_{k}$ denote the cofactor of the kth diagonal element of the Laplacian matrix of $(\mathcal{G}, A)$. Then the following identity holds:

$$
\sum_{k, h=1}^{n} c_{k} a_{k h} F_{k h}\left(x_{k}, x_{h}\right)=\sum_{\mathcal{Q} \in \mathbb{Q}} W(\mathcal{Q}) \sum_{(s, r) \in E\left(\mathcal{C}_{\mathcal{Q}}\right)} F_{r s}\left(x_{r}, x_{s}\right) .
$$

Here, for any $k, h \in \mathbb{L}, F_{k h}\left(x_{k}, x_{h}\right)$ is an arbitrary function, $\mathbb{Q}$ is the set of all spanning unicyclic graphs of $(\mathcal{G}, A), W(\mathcal{Q})$ is the weight of $\mathcal{Q}$, and $\mathcal{C}_{\mathcal{Q}}$ denotes the directed cycle of $\mathcal{Q}$. In particular, if $(\mathcal{G}, A)$ is strongly connected, then $c_{k}>0$ for $k \in \mathbb{L}$.

\subsection{Model formulations}

To begin with, we will establish CSTCS on a digraph $\mathcal{G}$ with $l(l \geq 2)$ vertices. We assume that the $k$ th vertex is described by

$$
\frac{\mathrm{d} x_{k}(t)}{\mathrm{d} t}=f_{k}\left(x_{k}(t), t\right), \quad t \geq 0,
$$

where $x_{k}=\left(x_{k_{1}}, x_{k_{2}}, \ldots, x_{k_{n_{k}}}\right)^{\mathrm{T}} \in \mathbb{R}^{n_{k}}$, and $f_{k}: \mathbb{R}^{n_{k}} \times \mathbb{R}_{+}^{1} \rightarrow \mathbb{R}^{n_{k}}$ is a continuous function.

Secondly, we take the influence of coupling structure into account. Let $a_{k h}(t)$ and $H_{k h}\left(x_{k}, x_{h}\right)$ be continuous functions, representing the time-varying coupling strength and coupling function of the $h$ th vertex to the $k$ th vertex, respectively. Here $a_{k h}(t) H_{k h}\left(x_{k}, x_{h}\right)=$ 0 if and only if there exists no coupling influence from the $h$ th vertex to the $k$ th vertex for any $t \geq 0$. Hence, for the $k$ th vertex, via replacing $f_{k}\left(x_{k}(t), t\right)$ by $f_{k}\left(x_{k}(t), t\right)+$ $\sum_{h=1}^{l} a_{k h}(t) H_{k h}\left(x_{k}(t), x_{h}(t)\right)$ we get the following CSTCS:

$$
\frac{\mathrm{d} x_{k}(t)}{\mathrm{d} t}=f_{k}\left(x_{k}(t), t\right)+\sum_{h=1}^{l} a_{k h}(t) H_{k h}\left(x_{k}(t), x_{h}(t)\right), \quad k \in \mathbb{L} .
$$

In the proofs of our main theorems, a Lyapunov function for system (3) is constructed by combining the Lyapunov functions of vertices with the coupling structure. In the sequel, the definition of a vertex Lyapunov function set is given as follows.

Definition 1 The set $\left\{V_{k}\left(x_{k}, t\right), k \in \mathbb{L}\right\}$ is called a vertex Lyapunov function set for system

(3) if the following conditions are satisfied for any $k \in \mathbb{L}$ and $t \geq 0$.

V1. There exist functions $V_{k}\left(x_{k}, t\right), b_{k h}(t)$ and $F_{k h}$ such that

$$
\begin{aligned}
\frac{\mathrm{d} V_{k}\left(x_{k}, t\right)}{\mathrm{d} t} & \triangleq \frac{\partial V_{k}\left(x_{k}, t\right)}{\partial t}+\frac{\partial V_{k}\left(x_{k}, t\right)}{\partial x_{k}}\left(f_{k}\left(x_{k}(t), t\right)+\sum_{h=1}^{l} a_{k h}(t) H_{k h}\left(x_{k}(t), x_{h}(t)\right)\right) \\
& \leq \sum_{h=1}^{l} b_{k h}(t) F_{k h}\left(x_{k}(t), x_{h}(t)\right)-\frac{d_{k}^{\prime}(t)}{d_{k}(t)} V_{k}\left(x_{k}(t), t\right),
\end{aligned}
$$

where $d_{k}(t)$ is the cofactor of the kth diagonal element of the Laplacian matrix of $\left(\mathcal{G},\left(b_{k h}(t)\right)_{l \times l}\right)$. 
V2. Along each directed cycle $\mathcal{C}$ of the weighted digraph $\left.\left(\mathcal{G},\left(b_{k h}(t)\right)\right)_{l \times l}\right)$, we have

$$
\sum_{(h, k) \in E(\mathcal{C})} F_{k h}\left(x_{k}(t), x_{h}(t)\right) \leq 0
$$

for all $x_{k} \in \mathbb{R}^{n_{k}}, x_{h} \in \mathbb{R}^{n_{h}}$.

We always suppose that all assumptions as regards the existence and uniqueness in Theorems 1.1 and 3.1 in [31] are fulfilled, so system (3) has a unique global solution for any given initial value $x(0)=x_{0}$, and we here denote the solution by $x\left(t ; x_{0}\right)$. Assume furthermore that $f_{k}(0, t)=0$ and $H_{k h}(0,0)=0$. Then system (3) has a trivial solution $x(t) \equiv 0$.

\section{Global stability analysis for CSTCS}

In this section, we investigate the stability of the trivial solution of system (3). Based on the graph theory and the Lyapunov method, we shall establish a theoretical framework for constructing a global Lyapunov function of system (3). The method used in the proof of main results is motivated by $[13,14]$.

Throughout this section, we always assume that $\left(\mathcal{G},\left(b_{k h}(t)\right)_{l \times l}\right)$ is strongly connected for any $t \geq 0$.

Theorem 1 Suppose that system (3) admits a vertex Lyapunov functions set $\left\{V_{k}\left(x_{k}, t\right)\right.$, $k \in \mathbb{L}\}$. Then

$$
V(x, t)=\sum_{k=1}^{l} d_{k}(t) V_{k}\left(x_{k}, t\right)
$$

satisfies $\dot{V}(x(t), t) \leq 0$ for $t \geq 0$. In other words, it is a Lyapunov function of system (3).

Proof By using (5) and condition V1, we can derive that

$$
\begin{aligned}
\frac{\mathrm{d} V(x(t), t)}{\mathrm{d} t}= & \sum_{k=1}^{l} \frac{\mathrm{d}\left(d_{k}(t) V_{k}\left(x_{k}(t), t\right)\right)}{\mathrm{d} t} \\
\leq & \sum_{k=1}^{l}\left[d_{k}^{\prime}(t) V_{k}\left(x_{k}(t), t\right)+d_{k}(t)\left(\sum_{h=1}^{l} b_{k h}(t) F_{k h}\left(x_{k}(t), x_{h}(t)\right)\right.\right. \\
& \left.\left.-\frac{d_{k}^{\prime}(t)}{d_{k}(t)} V_{k}\left(x_{k}(t), t\right)\right)\right] \\
= & \sum_{k=1}^{l} \sum_{h=1}^{l} d_{k}(t) b_{k h}(t) F_{k h}\left(x_{k}(t), x_{h}(t)\right) .
\end{aligned}
$$

By Lemma 1, we have

$$
\sum_{k=1}^{l} \sum_{h=1}^{l} d_{k}(t) b_{k h}(t) F_{k h}\left(x_{k}(t), x_{h}(t)\right)=\sum_{\mathcal{Q}(t) \in \mathbb{Q}(t)} W(\mathcal{Q}(t)) \sum_{(s, r) \in E\left(\mathcal{C}_{\mathcal{Q}(t)}\right)} F_{r s}\left(x_{r}(t), x_{s}(t)\right),
$$


where $\mathbb{Q}(t)$ is the set of all spanning unicyclic graph of $\left(\mathcal{G},\left(b_{k h}(t)\right)_{l \times l}\right), W(\mathcal{Q}(t))$ is the weight of $\mathcal{Q}(t)$, and $\mathcal{C}_{\mathcal{Q}(t)}$ denotes the directed cycle of $\mathcal{Q}(t)$. Then, making use of condition V2 and the fact that $W(\mathcal{Q}(t)) \geq 0$, we obtain

$$
\frac{\mathrm{d} V(x(t), t)}{\mathrm{d} t} \leq \sum_{\mathcal{Q}(t) \in \mathbb{Q}(t)} W(\mathcal{Q}(t)) \sum_{(s, r) \in E\left(\mathcal{C}_{\mathcal{Q}(t)}\right)} F_{r s}\left(x_{r}(t), x_{s}(t)\right) \leq 0 .
$$

The proof is complete.

With the help of some properties in graph theory, now some other simple conditions are discussed. Note that if $\left(\mathcal{G},\left(b_{k h}(t)\right)_{l \times l}\right)$ is balanced for any $t \geq 0$, then

$$
\begin{aligned}
\sum_{k=1}^{l} & \sum_{h=1}^{l} d_{k}(t) b_{k h}(t) F_{k h}\left(x_{k}(t), x_{h}(t)\right) \\
\quad= & \frac{1}{2} \sum_{\mathcal{Q}(t) \in \mathbb{Q}(t)} W(\mathcal{Q}(t)) \sum_{(h, k) \in E\left(\mathcal{C}_{\mathcal{Q}(t))}\right.}\left[F_{k h}\left(x_{k}(t), x_{h}(t)\right)+F_{h k}\left(x_{h}(t), x_{k}(t)\right)\right] .
\end{aligned}
$$

In this case, (4) can be replaced by

$$
\sum_{(k, h) \in E\left(\mathcal{C}_{\mathcal{Q}(t)}\right)}\left[F_{k h}\left(x_{k}(t), x_{h}(t)\right)+F_{h k}\left(x_{h}(t), x_{k}(t)\right)\right] \leq 0
$$

Based on this, a corollary is showed below.

Corollary 1 If $\left(\mathcal{G},\left(b_{k h}(t)\right)_{l \times l}\right)$ is balanced for any $t \geq 0$, the conclusion of Theorem 1 holds if (4) is replaced by (7).

Furthermore, if for every $F_{k h}\left(x_{k}(t), x_{h}(t)\right)$ there exist functions $P_{k}\left(x_{k}(t)\right)$ and $P_{h}\left(x_{h}(t)\right)$, such that

$$
F_{k h}\left(x_{k}(t), x_{h}(t)\right) \leq P_{k}\left(x_{k}(t)\right)-P_{h}\left(x_{h}(t)\right)
$$

then (4) follows naturally. Thus we obtain one more corollary below.

Corollary 2 If $\left(\mathcal{G},\left(b_{k h}(t)\right)_{l \times l}\right)$ is balanced for any $t \geq 0$, the conclusion of Theorem 1 holds if (4) is replaced by (8).

Remark 1 In Theorem 1, we apply the graph theory and the Lyapunov method to prove the stability of system (3). Recently, the stability problem for coupled systems has been widely studied. In [14], Li et al. used graph theory to explore the global-stability problem for coupled systems of differential equations on networks. In this work, if $a_{k h}(t)=a_{k h}$, our conclusion will be consistent with the Theorem 3.1 in [14]. Hence, our work is a generalization of the previous studies of CSs with time-invariant structure.

Remark 2 The stability result is based on the vertex Lyapunov functions set in Theorem 1 . In practical applications, since finding a suitable Lyapunov function is quite difficult, the stability criterion in Theorem 1 is not very convenient to be verified for a given system. 
Thus it prompts us to establish a coefficient-type criterion for the stability analysis of system (3).

Theorem 2 Let $p \geq 2$. The trivial solution of system (3) is stable if the following conditions hold for any $k, h \in \mathbb{L}$ and $t \geq 0$.

A1. There is a constant $\alpha_{k}>0$ such that

$$
x_{k}^{\mathrm{T}} f_{k}\left(x_{k}, t\right) \leq-\alpha_{k}\left|x_{k}\right|^{2} .
$$

A2. There exists a constant $A_{k h}$ such that

$$
\left|H_{k h}\left(x_{k}, x_{h}\right)\right| \leq A_{k h}\left(\left|x_{k}\right|+\left|x_{h}\right|\right) \text {. }
$$

A3. The digraph $(\mathcal{G}, H(t))$, where $H(t)=\left(A_{k h} a_{k h}(t)\right)_{l \times l}$, is strongly connected and

$$
-\alpha_{k} p+2 p \sum_{h=1}^{l} A_{k h} a_{k h}(t) \leq-\frac{g_{k}^{\prime}(t)}{g_{k}(t)},
$$

where $g_{k}(t)$ is the cofactor of the kth diagonal element of the Laplacian matrix of $(\mathcal{G}, H(t))$.

Proof For any $k \in \mathbb{L}$, define a function $V_{k}\left(x_{k}, t\right)=\left|x_{k}\right|^{p}$. Making use of condition A1, we get

$$
\begin{aligned}
& \frac{\mathrm{d} V_{k}\left(x_{k}(t), t\right)}{\mathrm{d} t} \\
& \quad=p\left|x_{k}(t)\right|^{p-2} x_{k}^{\mathrm{T}}(t)\left[f_{k}\left(x_{k}(t), t\right)+\sum_{h=1}^{l} a_{k h}(t) H_{k h}\left(x_{k}(t), x_{h}(t)\right)\right] \\
& \quad \leq p\left[-\alpha_{k}\left|x_{k}(t)\right|^{p}+\sum_{h=1}^{l} a_{k h}(t)\left|x_{k}(t)\right|^{p-1}\left|H_{k h}\left(x_{k}(t), x_{h}(t)\right)\right|\right] .
\end{aligned}
$$

By using condition A2 and the inequality (see [32], p.52)

$$
|a|^{p}|b|^{q} \leq \frac{p}{p+q}|a|^{(p+q)}+\frac{q}{p+q}|b|^{(p+q)}
$$

in which $p, q>0$, we have

$$
\begin{aligned}
& \sum_{h=1}^{l} a_{k h}(t)\left|x_{k}(t)\right|^{p-1}\left|H_{k h}\left(x_{k}(t), x_{h}(t)\right)\right| \\
& \quad \leq \frac{2 p-1}{p} \sum_{h=1}^{l} A_{k h} a_{k h}(t)\left|x_{k}(t)\right|^{p}+\frac{1}{p} \sum_{h=1}^{l} A_{k h} a_{k h}(t)\left|x_{h}(t)\right|^{p} .
\end{aligned}
$$


Substituting it into (10) yields

$$
\begin{aligned}
& \frac{\mathrm{d} V_{k}\left(x_{k}(t), t\right)}{\mathrm{d} t} \\
& \leq p\left[-\alpha_{k}\left|x_{k}(t)\right|^{p}+\sum_{h=1}^{l} a_{k h}(t)\left|x_{k}(t)\right|^{p-1}\left|H_{k h}\left(x_{k}(t), x_{h}(t)\right)\right|\right] \\
& \leq\left(-\alpha_{k} p+(2 p-1) \sum_{h=1}^{l} A_{k h} a_{k h}(t)\right)\left|x_{k}(t)\right|^{p}+\sum_{h=1}^{l} A_{k h} a_{k h}(t)\left|x_{h}(t)\right|^{p} \\
& \quad\left(-\alpha_{k} p+2 p \sum_{h=1}^{l} A_{k h} a_{k h}(t)\right)\left|x_{k}(t)\right|^{p}+\sum_{h=1}^{l} A_{k h} a_{k h}(t) F_{k h}\left(x_{k}(t), x_{h}(t)\right) \\
& \leq-\frac{g_{k}^{\prime}(t)}{g_{k}(t)} V_{k}\left(x_{k}(t), t\right)+\sum_{h=1}^{l} A_{k h} a_{k h}(t) F_{k h}\left(x_{k}(t), x_{h}(t)\right),
\end{aligned}
$$

where $F_{k h}\left(x_{k}(t), x_{h}(t)\right)=\left|x_{h}(t)\right|^{p}-\left|x_{k}(t)\right|^{p}$. Therefore, we verify the existence of a vertex Lyapunov functions set $\left\{V_{k}\left(x_{k}, t\right), k \in \mathbb{L}\right\}$ for system (3). Then we see that the trivial solution of system (3) is stable, which completes this proof.

Remark 3 In Theorem 2, considering condition A3 one needs to compute the derivative of determinant. Computing the derivative of high-order determinant is difficult. Thus the condition of Theorem 2 is not easy to verify. In Theorems 3, 4, we will use conditions that are described by the coefficients $a_{k h}(t)$ to study the stability of system (3), which are easier to verify.

Theorem 3 When $a_{k h}^{\prime}(t) \leq 0$ for all $k, h \in \mathbb{L}$, the conclusion of Theorem 2 holds if (9) is replaced by

$$
-\alpha_{k} p+2 p \sum_{h=1}^{l} A_{k h} a_{k h}(t) \leq 0 .
$$

Proof By the definition of $H(t)$, let $B_{i j}=A_{i j} a_{i j}(t)$ and we can obtain

$g_{k}(t)$

$$
=\left|\begin{array}{ccccccc}
\sum_{i \neq 1}^{l} B_{1 i} & -B_{12} & \ldots & -B_{1, k-1} & -B_{1, k+1} & \ldots & -B_{1 l} \\
-B_{21} & \sum_{i \neq 2}^{l} B_{2 i} & \ldots & -B_{2, k-1} & -B_{2, k+1} & \ldots & -B_{2 l} \\
\vdots & \vdots & \vdots & \vdots & \vdots & \vdots & \vdots \\
-B_{k-1,1} & -B_{k-1,2} & \ldots & \sum_{i \neq k-1}^{l} B_{k-1, i} & -B_{k-1, k+1} & \ldots & -B_{k-1, l} \\
-B_{k+1,1} & -B_{k+1,2}= & \ldots & -B_{k+1, k-1} & \sum_{i \neq k+1}^{l} B_{k+1, i} & \ldots & -B_{k+1, l} \\
\vdots & \vdots & \vdots & \vdots & \vdots & \vdots & \vdots \\
-B_{l 1} & -B_{l 2} & \ldots & -B_{l, k-1} & -B_{l, k+1} & \ldots & \sum_{i \neq l}^{l} B_{l i}
\end{array}\right| .
$$


Because the digraph is strongly connected and $a_{k h}^{\prime}(t) \leq 0$, we can obtain $g_{k}^{\prime}(t) \leq 0$. Furthermore, we can compute that

$$
g_{k}^{\prime}(t)=-\sum_{j \neq k}\left|\begin{array}{cccccc}
\vdots & \vdots & \vdots & \vdots & \vdots & \vdots \\
A_{j 1} a_{j 1}^{\prime}(t) & A_{j 2} a_{j 2}^{\prime}(t) & \cdots & \sum_{j \neq i}^{l}\left(-A_{j i} a_{j i}^{\prime}(t)\right) & \cdots & A_{j l} a_{j l}^{\prime}(t) \\
\vdots & \vdots & \vdots & \vdots & \vdots & \vdots
\end{array}\right| .
$$

Because $a_{k h}^{\prime}(t) \leq 0$, we have $g_{k}^{\prime}(t) \leq 0$. Thus we obtain

$$
-\frac{g_{k}^{\prime}(t)}{g_{k}(t)} \geq 0
$$

Consequently, it easily follows that

$$
-\alpha_{k} p+2 p \sum_{h=1}^{l} A_{k h} a_{k h}(t) \leq-\frac{g_{k}^{\prime}(t)}{g_{k}(t)} .
$$

Then the condition A3 is satisfied. Thus we have verified all conditions in Theorem 2. This completes the proof.

Theorem 4 When $a_{k h}(t)=a_{k}(t), k, h \in \mathbb{L}$, the conclusion of Theorem 2 holds if (9) is replaced by

$$
-\alpha_{k} p+2 p \sum_{h=1}^{l} A_{k h} a_{k}(t)+\sum_{i=1, i \neq k}^{l} \frac{a_{i}^{\prime}(t)}{a_{i}(t)} \leq 0 .
$$

Proof By the definition of $H(t)$, we have

$g_{k}(t)$

$$
\begin{aligned}
& =\prod_{i=1, i \neq k}^{l} a_{i}(t)\left|\begin{array}{cccccc}
\sum_{i \neq 1}^{l} A_{1 i} & \cdots & -A_{1, k-1} & -A_{1, k+1} & \cdots & -A_{1 l} \\
-A_{21} & \cdots & -A_{2, k-1} & -A_{2, k+1} & \cdots & -A_{2 l} \\
\vdots & \vdots & \vdots & \vdots & \vdots & \vdots \\
-A_{k-1,1} & \cdots & \sum_{i \neq k-1}^{l} A_{k-1, i} & -A_{k-1, k-1} & \cdots & -A_{k-1, l} \\
-A_{k+1,1} & \cdots & -A_{k+1, k-1} & \sum_{i \neq k+1}^{l} A_{k+1, i} & \cdots & -A_{k+1, l} \\
\vdots & \vdots & \vdots & \vdots & \vdots & \vdots \\
-A_{l 1} & \cdots & -A_{l, k-1} & -A_{l, k+1} & \cdots & \sum_{i \neq l}^{l} A_{l i}
\end{array}\right| \\
& \triangleq \prod_{i=1, i \neq k}^{l} a_{i}(t) \cdot G,
\end{aligned}
$$


where

$$
G=\left|\begin{array}{ccccccc}
\sum_{i \neq 1}^{l} A_{1 i} & -A_{12} & \cdots & -A_{1, k-1} & -A_{1, k+1} & \cdots & -A_{1 l} \\
-A_{21} & \sum_{i \neq 2}^{l} A_{2 i} & \cdots & -A_{2, k-1} & -A_{2, k+1} & \cdots & -A_{2 l} \\
\vdots & \vdots & \vdots & \vdots & \vdots & \vdots & \vdots \\
-A_{k-1,1} & -A_{k-1,2} & \cdots & \sum_{i \neq k-1}^{l} A_{k-1, i} & -A_{k-1, k-1} & \cdots & -A_{k-1, l} \\
-A_{k+1,1} & -A_{k+1,2} & \cdots & -A_{k+1, k-1} & \sum_{i \neq k+1}^{l} A_{k+1, i} & \cdots & -A_{k+1, l} \\
\vdots & \vdots & \vdots & \vdots & \vdots & \vdots & \vdots \\
-A_{l 1} & -A_{l 2} & \cdots & -A_{l, k-1} & -A_{l, k+1} & \cdots & \sum_{i \neq l}^{l} A_{l i}
\end{array}\right| .
$$

Then it is easy to get

$$
g_{k}^{\prime}(t)=\sum_{i=1, i \neq k}^{l} a_{1}(t) \cdot a_{2}(t) \cdots a_{i}^{\prime}(t) \cdots a_{l}(t) \cdot G .
$$

Thus

$$
\frac{g_{k}^{\prime}(t)}{g_{k}(t)}=\sum_{i=1, i \neq k}^{l} \frac{a_{i}^{\prime}(t)}{a_{i}(t)} .
$$

Therefore, condition A3 holds. Then the proof is complete.

\section{An application to the predator-prey model with time-varying dispersal}

In this section, we consider a predator-prey model, in which the dispersal rate of preys among $l(l \geq 2)$ patches is time-varying, the details are as follows:

$$
\left\{\begin{array}{l}
\frac{\mathrm{d} x_{k}}{\mathrm{~d} t}=x_{k}\left(r_{k}-b_{k} x_{k}-e_{k} y_{k}\right)+\sum_{h=1}^{l} a_{k h}(t)\left(x_{h}-\alpha_{k h} x_{k}\right), \\
\frac{\mathrm{d} y_{k}}{\mathrm{~d} t}=y_{k}\left(-\gamma_{k}-\delta_{k} y_{k}+\varepsilon_{k} x_{k}\right), \quad k \in \mathbb{L},
\end{array}\right.
$$

where $x_{k}$ and $y_{k}$ denote the densities of preys and predators on the patch $k$, respectively. The parameters in the system (11) are nonnegative constants, and $e_{k}, \varepsilon_{k}, \delta_{k}$ and $b_{k}$ are positive. The notations and useful definitions in this section are as follows:

$r_{k}: \quad$ the intrinsic growth rate of the preys in the absence of predation in patch $k$,

$\gamma_{k}$ : the death rate of the predators in patch $k$,

$b_{k}$ : the intra-specific competition rate of the preys in patch $k$,

$\delta_{k}$ : the intra-specific competition rate of the predators in patch $k$,

$\varepsilon_{k} x_{k}$ : the proportion of preys which are eaten and become predators,

$e_{k}: \quad$ the predator response function for the predator with respect to that particular prey in the $k$ th patch.

Function $a_{k h}(t) \geq 0$ is the dispersal rate of preys from patch $h$ to patch $k$. Constant $\alpha_{k h} \geq$ 0 is a boundary condition in the continuous diffusion case.

Remark 4 In existing literature, many researchers have investigated the predator-prey model with time-invariant dispersal (see [21,33] and the references therein). However, to our knowledge, there is little work as regards the predator-prey model with time-varying 
dispersal. In this paper, if $a_{k h}(t)=a_{k h}$, system (11) turns into system (6.1) in [14]. Hence, system (11) is much more general since it considers a dispersal rate of preys which is not constant but time-varying.

Remark 5 If we simplify system (11), in other words, if we let $r_{k}=r, b_{k}=b, e_{k}=e, \gamma_{k}=\gamma$, $\delta_{k}=\delta, \varepsilon_{k}=\varepsilon$, and $a_{k h}(t)=\alpha_{k h}=1$, then it is trivial to see that system (11) has a unique equilibrium: $x^{*}=\frac{\delta r+e \gamma}{\delta b+e \varepsilon}, y^{*}=\frac{\varepsilon r-b \gamma}{\delta b+e \varepsilon}$. If condition $\varepsilon r>b \gamma$ is satisfied, we can guarantee that $\left(x^{*}, y^{*}\right)^{\mathrm{T}}$ is a positive equilibrium. So it is reasonable to assume that there exists a positive equilibrium $E^{*}=\left(x_{1}^{*}, y_{1}^{*}, \ldots, x_{l}^{*}, y_{l}^{*}\right)^{\mathrm{T}}$ for system (11).

Let $D(t)=\left(a_{k h}(t) \varepsilon_{k} x_{h}^{*}\right)_{l \times l}$, and $l_{k}(t)$ be the cofactor of the $k$ th diagonal element of the Laplacian matrix of $(\mathcal{G}, D(t))$, respectively.

Theorem 5 For any $t \geq 0$, assume that the digraph $(\mathcal{G}, D(t))$ is strongly connected and the following inequality holds:

$$
l_{k}^{\prime}(t) \leq 0, \quad k \in \mathbb{L} .
$$

Then, as long as a positive equilibrium $E^{*}$ exists, it is unique and globally asymptotically stable in the positive cone $\mathbb{R}_{+}^{2 l}$.

Proof Consider the Lyapunov functions

$$
V_{1}^{(k)}\left(x_{k}\right)=\varepsilon_{k}\left(x_{k}-x_{k}^{*}-x_{k}^{*} \ln \frac{x_{k}}{x_{k}^{*}}\right)
$$

and

$$
V_{2}^{(k)}\left(y_{k}\right)=e_{k}\left(y_{k}-y_{k}^{*}-y_{k}^{*} \ln \frac{y_{k}}{y_{k}^{*}}\right), \quad k \in \mathbb{L} .
$$

Making use of system (11), we get

$$
\begin{aligned}
\frac{\mathrm{d} V_{1}^{(k)}\left(x_{k}\right)}{\mathrm{d} t} & \varepsilon_{k}\left(1-\frac{x_{k}^{*}}{x_{k}}\right)\left[x_{k}\left(r_{k}-b_{k} x_{k}-e_{k} y_{k}\right)+\sum_{h=1}^{l} a_{k h}(t)\left(x_{h}-\alpha_{k h} x_{k}\right)\right] \\
= & \varepsilon_{k}\left(x_{k}-x_{k}^{*}\right)\left[-b_{k}\left(x_{k}-x_{k}^{*}\right)-e_{k}\left(y_{k}-y_{k}^{*}\right)+\sum_{h=1}^{l} a_{k h}(t)\left(\frac{x_{h}}{x_{k}}-\frac{x_{h}^{*}}{x_{k}^{*}}\right)\right] \\
= & -\varepsilon_{k} b_{k}\left(x_{k}-x_{k}^{*}\right)^{2}-\varepsilon_{k} e_{k}\left(x_{k}-x_{k}^{*}\right)\left(y_{k}-y_{k}^{*}\right) \\
& +\sum_{h=1}^{l} \varepsilon_{k} x_{h}^{*} a_{k h}(t)\left(\frac{x_{h}}{x_{h}^{*}}-\frac{x_{k}}{x_{k}^{*}}-\frac{x_{k}^{*} x_{h}}{x_{k} x_{h}^{*}}+1\right) \\
\triangleq & -\varepsilon_{k} b_{k}\left(x_{k}-x_{k}^{*}\right)^{2}-\varepsilon_{k} e_{k}\left(x_{k}-x_{k}^{*}\right)\left(y_{k}-y_{k}^{*}\right)+\sum_{h=1}^{l} \theta_{k h}(t) F_{k h}\left(x_{k}, x_{h}\right),
\end{aligned}
$$


where $\theta_{k h}(t)=\varepsilon_{k} x_{h}^{*} a_{k h}(t)$ and $F_{k h}\left(x_{k}, x_{h}\right)=x_{h} / x_{h}^{*}-x_{k} / x_{k}^{*}-x_{k}^{*} x_{h} /\left(x_{k} x_{h}^{*}\right)+1$. Similarly,

$$
\begin{aligned}
& \frac{\mathrm{d} V_{2}^{(k)}\left(y_{k}\right)}{\mathrm{d} t} \\
& \quad=e_{k} y_{k}\left(1-\frac{y_{k}^{*}}{y_{k}}\right)\left(-\gamma_{k}-\delta_{k} y_{k}+\varepsilon_{k} x_{k}\right) \\
& \quad=e_{k}\left(y_{k}-y_{k}^{*}\right)\left[\varepsilon_{k}\left(x_{k}-x_{k}^{*}\right)-\delta_{k}\left(y_{k}-y_{k}^{*}\right)\right] .
\end{aligned}
$$

Let

$$
V=\sum_{k=1}^{l} l_{k}(t)\left(V_{1}^{(k)}\left(x_{k}\right)+V_{2}^{(k)}\left(y_{k}\right)\right) .
$$

By (12), (15) and (16), we have

$$
\begin{aligned}
\frac{\mathrm{d} V}{\mathrm{~d} t}= & \sum_{k=1}^{l}\left[l_{k}(t)\left(\frac{\mathrm{d} V_{1}^{(k)}\left(x_{k}\right)}{\mathrm{d} t}+\frac{\mathrm{d} V_{2}^{(k)}\left(y_{k}\right)}{\mathrm{d} t}\right)+l_{k}^{\prime}(t)\left(V_{1}^{(k)}\left(x_{k}\right)+V_{2}^{(k)}\left(y_{k}\right)\right)\right] \\
= & \sum_{k=1}^{l}\left[l_{k}(t)\left(-\varepsilon_{k} b_{k}\left(x_{k}-x_{k}^{*}\right)^{2}+\sum_{h=1}^{l} \theta_{k h}(t) F_{k h}\left(x_{k}, x_{h}\right)-e_{k} \delta_{k}\left(y_{k}-y_{k}^{*}\right)^{2}\right)\right. \\
& \left.+l_{k}^{\prime}(t)\left(V_{1}^{(k)}\left(x_{k}\right)+V_{2}^{(k)}\left(y_{k}\right)\right)\right] \\
= & \sum_{k=1}^{l}\left[-\varepsilon_{k} b_{k} l_{k}(t)\left(x_{k}-x_{k}^{*}\right)^{2}+\varepsilon_{k} l_{k}^{\prime}(t)\left(x_{k}-x_{k}^{*}-x_{k}^{*} \ln \frac{x_{k}}{x_{k}^{*}}\right)\right. \\
& \left.-e_{k} \delta_{k} l_{k}(t)\left(y_{k}-y_{k}^{*}\right)^{2}+e_{k} l_{k}^{\prime}(t)\left(y_{k}-y_{k}^{*}-y_{k}^{*} \ln \frac{y_{k}}{y_{k}^{*}}\right)\right] \\
& +\sum_{k=1}^{l} \sum_{h=1}^{l} l_{k}(t) \theta_{k h}(t) F_{k h}\left(x_{k}, x_{h}\right) \\
\leq & \sum_{k=1}^{l} \sum_{h=1}^{l} l_{k}(t) \theta_{k h}(t) F_{k h}\left(x_{k}, x_{h}\right) \\
\leq & 0 .
\end{aligned}
$$

Since $(\mathcal{G}, D(t))$ is strongly connected for any $t \geq 0, l_{k}(t)>0$ for $k \in \mathbb{L}$. Note the fact that

$$
F_{k h}\left(x_{k}, x_{h}\right)=\frac{x_{h}}{x_{h}^{*}}-\frac{x_{k}}{x_{k}^{*}}-\frac{x_{k}^{*} x_{h}}{x_{k} x_{h}^{*}}+1 \leq\left(-\frac{x_{k}}{x_{k}^{*}}+\ln \frac{x_{k}}{x_{k}^{*}}\right)-\left(-\frac{x_{h}}{x_{h}^{*}}+\ln \frac{x_{h}}{x_{h}^{*}}\right)
$$

and the quality holds if and only if $x_{k}^{*} x_{h} / x_{k} x_{h}^{*}=1$. Hence, we have $\dot{V}=0$ if and only if $-\varepsilon_{k} b_{k} l_{k}(t)\left(x_{k}-x_{k}^{*}\right)^{2}=0, \varepsilon_{k} l_{k}^{\prime}(t)\left(x_{k}-x_{k}^{*}-x_{k}^{*} \ln \frac{x_{k}}{x_{k}^{*}}\right)=0,-e_{k} \delta_{k} l_{k}(t)\left(y_{k}-y_{k}^{*}\right)^{2}=0, e_{k} l_{k}^{\prime}(t)\left(y_{k}-\right.$ $\left.y_{k}^{*}-y_{k}^{*} \ln \frac{y_{k}}{y_{k}^{*}}\right)=0$ and $x_{k}^{*} x_{h} / x_{k} x_{h}^{*}=1$ for all $k, h \in \mathbb{L}$. Since $\varepsilon_{k}, b_{k}, e_{k}, \delta_{k}>0$ and $l_{k}(t)>0, t \geq 0$, we have $x_{k}=x_{k}^{*}, y_{k}=y_{k}^{*}$. And in view of the fact that $x_{k}^{*} x_{h}=x_{h}^{*} x_{k}$ for all $k, h \in \mathbb{L}$, one can 
see that $x_{h}=x_{h}^{*}$ for $h \in \mathbb{L}$. Then

$$
\left\{E \mid \frac{\mathrm{d} V}{\mathrm{~d} t}=0\right\}=\left\{E^{*}\right\}
$$

Therefore, by the LaSalle invariance principle, $E^{*}$ is globally asymptotically stable. This also implies that $E^{*}$ is unique in $\mathbb{R}_{+}^{2 l}$.

Based on Theorem 5 , by using the dispersal rate of preys $a_{k h}(t), k, h \in \mathbb{L}$, we can get two more simple stability criteria for system (11).

Theorem 6 For any $t \geq 0$, let $(\mathcal{G}, D(t))$ be strongly connected. If $a_{k h}^{\prime}(t) \leq 0$, then as long as a positive equilibrium $E^{*}$ exists, it is unique and globally asymptotically stable in the positive cone $\mathbb{R}_{+}^{2 l}$.

Proof In view of $\varepsilon_{k}>0$ and $x_{h}^{*}>0$, we have

$$
\left(a_{k h}(t) \varepsilon_{k} x_{h}^{*}\right)^{\prime}=a_{k h}^{\prime}(t) \varepsilon_{k} x_{h}^{*} \leq 0 .
$$

Then we can get $l_{k}^{\prime}(t) \leq 0$, and the proof is similar to the proof of $g_{k}^{\prime}(t) \leq 0$ in Theorem 3 , here, we omit it. This means that the conditions of Theorem 5 could be achieved. This completes the whole proof.

Theorem 7 For any $t \geq 0$, let $(\mathcal{G}, D(t))$ be strongly connected. When $a_{k h}(t)=a_{k}(t), k, h \in \mathbb{L}$, the conclusion of Theorem 6 holds if

$$
\sum_{i=1, i \neq k}^{l} \frac{a_{i}^{\prime}(t)}{a_{i}(t)} \leq 0 .
$$

Proof Similar to the proof of Theorem 4, we get

$$
l_{k}^{\prime}(t)=\sum_{i=1, i \neq k}^{l} l_{k}(t) \frac{a_{i}^{\prime}(t)}{a_{i}(t)} .
$$

Using $\sum_{i=1, i \neq k}^{l} \frac{a_{i}^{\prime}(t)}{a_{i}(t)} \leq 0$ and $l_{k}(t)>0$ one then sees that

$$
\begin{aligned}
\sum_{k=1}^{l} & {\left[-\varepsilon_{k} b_{k} l_{k}(t)\left(x_{k}-x_{k}^{*}\right)^{2}+\varepsilon_{k} l_{k}^{\prime}(t)\left(x_{k}-x_{k}^{*}-x_{k}^{*} \ln \frac{x_{k}}{x_{k}^{*}}\right)-e_{k} \delta_{k} l_{k}\left(y_{k}-y_{k}^{*}\right)^{2}\right.} \\
& \left.+e_{k} l_{k}^{\prime}(t)\left(y_{k}-y_{k}^{*}-y_{k}^{*} \ln \frac{y_{k}}{y_{k}^{*}}\right)\right] \\
= & \sum_{k=1}^{l} l_{k}(t)\left[-\varepsilon_{k} b_{k}\left(x_{k}-x_{k}^{*}\right)^{2}+\varepsilon_{k} \sum_{i=1, i \neq k}^{l} \frac{a_{i}^{\prime}(t)}{a_{i}(t)}\left(x_{k}-x_{k}^{*}-x_{k}^{*} \ln \frac{x_{k}}{x_{k}^{*}}\right)\right. \\
& \left.-e_{k} \delta_{k}\left(y_{k}-y_{k}^{*}\right)^{2}+e_{k} \sum_{i=1, i \neq k}^{l} \frac{a_{i}^{\prime}(t)}{a_{i}(t)}\left(y_{k}-y_{k}^{*}-y_{k}^{*} \ln \frac{y_{k}}{y_{k}^{*}}\right)\right]
\end{aligned}
$$

$\leq 0$. 
Furthermore, we can get

$$
\frac{\mathrm{d} V}{\mathrm{~d} t} \leq \sum_{k=1}^{l} \sum_{h=1}^{l} l_{k}(t) \theta_{k h}(t) F_{k h}\left(x_{k}, x_{h}\right) \leq 0 .
$$

It is now the same as the proof of Theorem 5. The proof is complete.

Remark 6 In Theorem 5, we have presented the stability criterion for a predator-prey model in which the dispersal rate $a_{k h}(t)$ is time-varying for any $k, h \in \mathbb{L}$. Li et al. [14] discussed a predator-prey model with time-invariant dispersal and achieved some results concerning the stability problem. In this paper, if $a_{k h}(t)=a_{k h}$, the conclusion of Theorem 6 is consistent with Theorem 6.1 in [14], which means our work generalizes a global-stability result from time-invariant dispersal to time-varying dispersal.

Remark 7 Biologically, time-varying dispersal, such as seasonal migration, is an extremely common phenomenon. As a result, the effect of time-varying dispersal on the species survival has been an important topic in population biology. This paper provides a possibility that researchers could use it to make the adjustment for the prey dispersal network to protect some species, which truly shows that the results in this paper are meaningful not only in theory but also in practice.

\section{Numerical simulations}

In this section, we will discuss a numerical example to illustrate the effectiveness and feasibility of Theorems 6 and 7.

Let $l=8$. Consider a predator-prey model in the form of system (11) with the following parameters:

$$
\begin{aligned}
& r_{k}=0.2, \quad b_{k}=0.4, \quad e_{k}=0.2, \quad \gamma_{k}=0.1, \\
& \delta_{k}=0.3, \quad \varepsilon_{k}=0.3, \quad k=1,2, \ldots, 4, \\
& r_{k}=0.15, \quad b_{k}=0.3, \quad e_{k}=0.15, \quad \gamma_{k}=\frac{1}{30}, \\
& \delta_{k}=0.5, \quad \varepsilon_{k}=0.2, \quad k=5,7, \ldots, 8, \\
& \alpha_{k h}=1, \quad(k, h=1,2, \ldots, 8) .
\end{aligned}
$$

Firstly, we can make a calculation to get $x_{k}^{*}=\frac{4}{9}, y_{k}^{*}=\frac{1}{9}, k=1,2, \ldots, 8$, which means that the positive equilibrium is

$$
E^{*}=\left(\frac{4}{9}, \frac{1}{9}, \frac{4}{9}, \frac{1}{9}, \ldots, \frac{4}{9}, \frac{1}{9}\right)_{16 \times 1}^{\mathrm{T}}
$$

The time-varying dispersal coefficients for preys are the followings: $a_{18}(t)=e^{-t}, a_{21}(t)=$ $t^{-1}, a_{32}(t)=e^{-2 t} a_{43}(t)=\left(\frac{1}{2}\right)^{t}, a_{54}(t)=e^{-3 t}, a_{65}(t)=e^{\sin t-t}, a_{76}(t)=\left(\frac{1}{3}\right)^{t}, a_{87}(t)=e^{-t^{2}}$. Ex cluding these, other dispersal coefficients $a_{k h}(t)=0, k, h=1,2, \ldots, 8$, we can easily get 
$a_{k h}^{\prime}(t) \leq 0, k, h=1,2, \ldots, 8$. And by the definition of $D(t)$, we can obtain the coefficientmatrix $D_{1}(t)$ for system (11) as follows:

$$
D_{1}(t)=\left(\begin{array}{cccccccc}
0 & 0 & 0 & 0 & 0 & 0 & 0 & \frac{2}{15} e^{-t} \\
\frac{2}{15} t^{-1} & 0 & 0 & 0 & 0 & 0 & 0 & 0 \\
0 & \frac{2}{15} e^{-2 t} & 0 & 0 & 0 & 0 & 0 & 0 \\
0 & 0 & \frac{2}{15}\left(\frac{1}{2}\right)^{t} & 0 & 0 & 0 & 0 & 0 \\
0 & 0 & 0 & \frac{4}{45} e^{-3 t} & 0 & 0 & 0 & 0 \\
0 & 0 & 0 & 0 & \frac{4}{45} e^{\sin t-t} & 0 & 0 & 0 \\
0 & 0 & 0 & 0 & 0 & \frac{4}{45}\left(\frac{1}{3}\right)^{t} & 0 & 0 \\
0 & 0 & 0 & 0 & 0 & 0 & \frac{4}{45} e^{-t^{2}} & 0
\end{array}\right) .
$$

Obviously, $\left(\mathcal{G}, D_{1}(t)\right)$ is strongly connected for any $t \geq 0$. Until now, all conditions in Theorem 6 have been checked. Hence, we can get the conclusion that the positive equilibrium $E^{*}$ is unique and globally asymptotically stable in the positive cone. The simulation result is shown in Figure 1.

When $a_{k h}(t)=a_{k}(t), k, h \in \mathbb{L}$, let the time-varying dispersal coefficients for preys be the following: $a_{1}(t)=0.01, a_{2}(t)=e^{\sin t}, a_{3}(t)=e^{-t}, a_{4}(t)=e^{-10 t}, a_{5}(t)=\frac{1}{t}, a_{6}(t)=\frac{1.1+\sin t}{10 t}, a_{7}(t)=$ $e^{\cos t}, a_{8}(t)=e^{-t}$. Coefficient-matrix $D_{2}(t)$ for system (11) can be obtained via the definition of $D(t)$ as follows: $D_{2}(t)=\left(a_{i j}\right)_{n \times n}$, where $a_{11}=a_{22}=a_{33}=a_{44}=a_{55}=a_{66}=a_{77}=a_{88}=0$, $a_{12}=a_{13}=a_{14}=a_{15}=a_{16}=a_{17}=a_{18}=\frac{1}{750}, a_{21}=a_{23}=a_{24}=a_{25}=a_{26}=a_{27}=a_{28}=\frac{2}{15} e^{\sin t}$, $a_{31}=a_{32}=a_{34}=a_{35}=a_{36}=a_{37}=a_{38}=\frac{2}{15} e^{-t}, a_{41}=a_{42}=a_{43}=a_{45}=a_{46}=a_{47}=a_{48}=$ $\frac{2}{15} e^{-10 t}, a_{51}=a_{52}=a_{53}=a_{54}=a_{56}=a_{57}=a_{58}=\frac{4}{45} \frac{1}{t}, a_{61}=a_{62}=a_{63}=a_{64}=a_{65}=a_{67}=$ $a_{68}=\frac{4}{45} \frac{1.1+\sin t}{10 t}, a_{71}=a_{72}=a_{73}=a_{74}=a_{75}=a_{76}=a_{78}=\frac{4}{45} e^{\cos t}, a_{81}=a_{82}=a_{83}=a_{84}=a_{85}=$

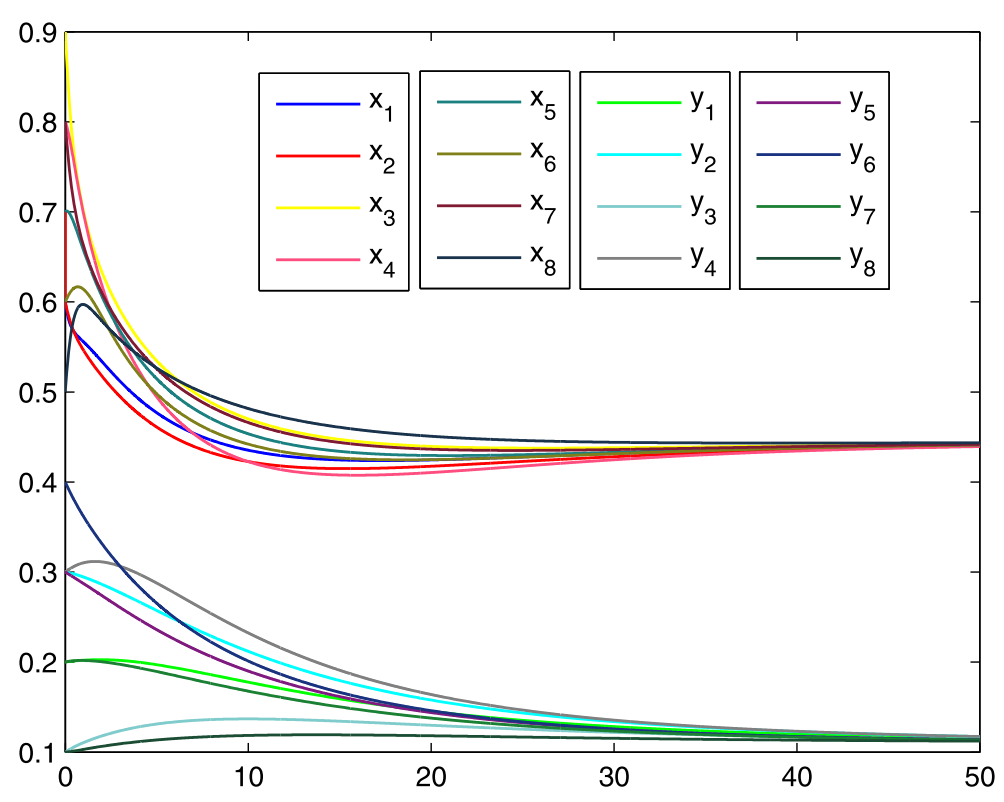

Figure 1 The solution of coupled predator-prey model with time-varying dispersal coefficient-matrix $D_{1}(t)$ when $I=8$. 


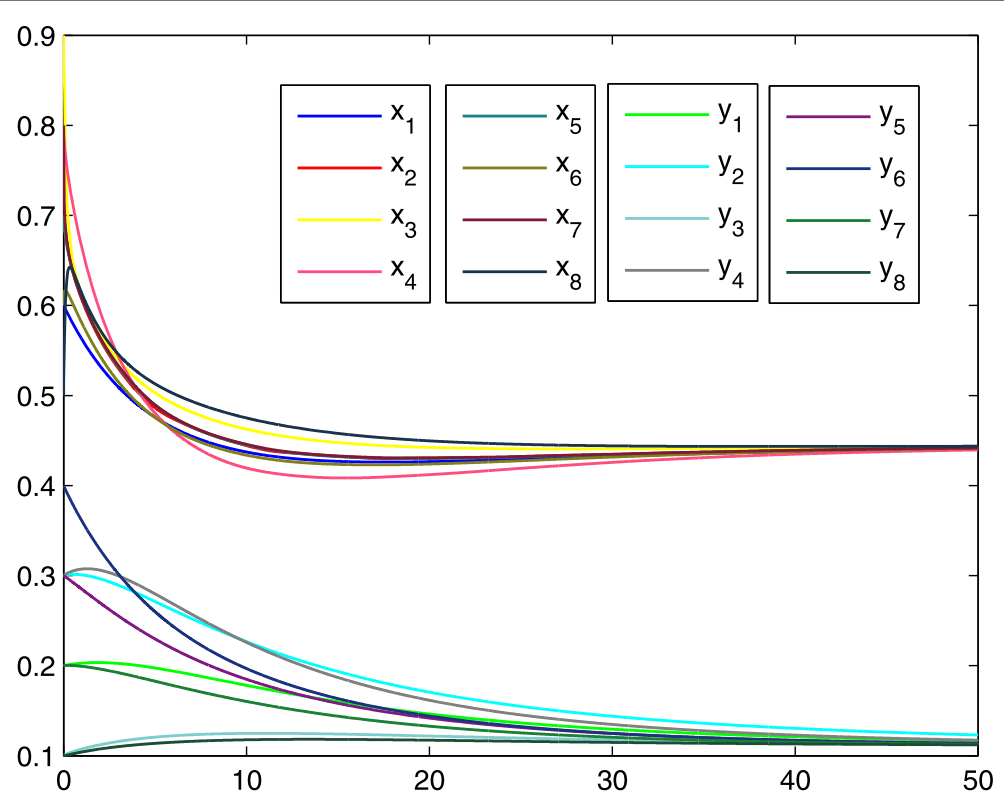

Figure 2 The solution of coupled predator-prey model with time-varying dispersal coefficient-matrix $D_{2}(t)$ when $I=8$.

$a_{86}=a_{87}=\frac{4}{45} e^{-t}$. And

$$
\begin{aligned}
\sum_{i=1, i \neq k}^{8} \frac{a_{i}^{\prime}(t)}{a_{i}(t)} & =\cos t-\sin t-12-\frac{1}{t}+\frac{t \cos t-1.1-\sin t}{t(1.1+\sin t)} \\
& \leq-2+\cos t-\sin t-\frac{2}{t}<0
\end{aligned}
$$

It is obvious that $\left(\mathcal{G}, D_{2}(t)\right)$ is strongly connected for any $t \geq 0$. We can easily check that all conditions of Theorem 7 are satisfied. And the equilibrium $E^{*}$ of system (11) with timevarying dispersal coefficient-matrix $D_{2}(t)$ is shown in Figure 2. In fact, we can clearly see from Figure 2 that the equilibrium $E^{*}$ is unique and globally asymptotically stable in the positive cone. The numerical simulation result verifies the effectiveness and feasibility of the proposed results.

Compared with the existing results on the stability of CSs $[13,14]$, our main contributions are as follows.

1. We generalize CSs with time-invariant coupling structure to CSTCS.

2. We first study the global stability for CSTCS by combining the graph theory with the Lyapunov method.

3. We apply these theoretical conclusions to a predator-prey model with time-varying dispersal. 
Authors' contributions

All authors contributed equally to the writing of this paper. All authors read and approved the final manuscript.

\section{Author details}

${ }_{1}^{1}$ College of Mathematics and Statistics, Shenzhen University, Shenzhen, 518060, P.R. China. ${ }^{2}$ School of Mathematical Sciences, University of Science and Technology of China, Hefei, 230026, P.R. China.

\section{Publisher's Note}

Springer Nature remains neutral with regard to jurisdictional claims in published maps and institutional affiliations.

Received: 27 November 2016 Accepted: 22 May 2017 Published online: 03 July 2017

\section{References}

1. Pecora, L, Carroll, T: Master stability functions for synchronized coupled systems. Syst. Control Lett. 80, 2109-2112 (1998)

2. Li, C, Xu, H, Liao, X, Yu, J: Synchronization in small-world oscillator networks with coupling delays. Physica A 335 , 359-364 (2004)

3. Zheng, S, Wang, S, Dong, G, Bi, S: Adaptive synchronization of two nonlinearly coupled complex dynamical networks with delayed coupling. Commun. Nonlinear Sci. Numer. Simul. 17, 284-291 (2012)

4. Yuan, Y, Zhao, X: Global stability for non-monotone delay equations (with application to a model of blood cell production). J. Differ. Equ. 252, 2189-12209 (2012)

5. May, R: Stability and Complexity in Model Ecosystems. Princeton University Press, Princeton (1973)

6. Thieme, H: Mathematics in Population Biology. Princeton University Press, Princeton (2003)

7. Bost, K, Cox, M, Payne, C: Structural and supportive changes in couples' family and friendship networks across the transition to parenthood. J. Marriage Fam. 64, 517-531 (2002)

8. Song, Q, Wang, Z: Stability analysis of impulsive stochastic Cohen-Grossberg neural networks with mixed time delays. Physica A 387, 3314-3326 (2008)

9. Lynch, J, York, R: Stability of mode locked states of coupled oscillator arrays. IEEE Trans. Circuits Syst. I, Fundam. Theory Appl. 42, 413-418 (1995)

10. Shu, H, Fan, D, Wei, J: Global stability of multi-group SEIR epidemic models with distributed delays and nonlinear transmission. Nonlinear Anal., Real World Appl. 13,1581-1592 (2012)

11. Muroya, Y, Enatsu, Y, Kuniya, T: Global stability for a multi-group SIRS epidemic model with varying population sizes. Nonlinear Anal., Real World Appl. 14, 1693-1704 (2013)

12. Guo, Y, Su, H, Ding, X, Wang, K: Global stochastic stability analysis for stochastic neural networks with infinite delay and Markovian switching. Appl. Math. Comput. 245, 53-65 (2014)

13. Guo, H, Li, MY, Shuai, Z: A graph-theoretic approach to the method of global Lyapunov functions. Proc. Am. Math Soc. 136, 2793-2802 (2008)

14. Li, YM, Shuai, Z: Global-stability problem for coupled systems of differential equations on networks. J. Differ. Equ. 248, $1-20(2010)$

15. Li, MY, Shuai, Z, Wang, C: Global stability of multi-group epidemic models with distributed delays. J. Math. Anal. Appl. 361, 38-47 (2010)

16. Sun, R: Global stability of the endemic equilibrium of multigroup SIR models with nonlinear incidence. Comput. Math. Appl. 60, 2286-2291 (2010)

17. Su, H, Qu, Y, Gao, S, Song, H, Wang, K: A model of feedback control system on network and its stability analysis. Commun. Nonlinear Sci. Numer. Simul. 18, 1822-1831 (2013)

18. Zhang, C, Li, W, Su, H, Wang, K: Asymptotic boundedness for stochastic coupled systems on networks with Markovian switching. Neurocomputing 136, 180-189 (2014)

19. Li, X, Fu, X: Stability analysis of stochastic functional differential equations with infinite delay and its application to recurrent neural networks. J. Comput. Appl. Math. 234, 407-417 (2010)

20. Li, T, Song, A, Fei, S: Global synchronization in arrays of coupled Lurie systems with both time-delay and hybrid coupling. Commun. Nonlinear Sci. Numer. Simul. 16, 10-20 (2011)

21. Zhang, C, Li, W, Wang, K: Graph-theoretic approach to stability of multi-group models with dispersal. Discrete Contin. Dyn. Syst., Ser. B 20, 259-280 (2015)

22. Song, H, Chen, D, Li, W, Qu, Y: Graph-theoretic approach to exponential synchronization of stochastic reaction-diffusion Cohen-Grossberg neural networks with time-varying delays. Neurocomputing 177, 179-187 (2016)

23. Wang, J, Zhang, X, Li, W: Periodic solutions of stochastic coupled systems on networks with periodic coefficients. Neurocomputing 205, 360-366 (2016)

24. Li, W, Su, H, Wang, K: Global stability analysis for stochastic coupled systems on networks. Automatica 47, 215-220 (2011)

25. Su, W, Chen, Y: Global asymptotic stability analysis for neutral stochastic neural networks with time-varying delays. Commun. Nonlinear Sci. Numer. Simul. 14, 1576-1581 (2009)

26. Kao, Y, Wang, C: Global stability analysis for stochastic coupled reaction-diffusion systems on networks. Nonlinear Anal., Real World Appl. 14, 1457-1465 (2013)

27. Cheng, P, Deng, F: Global exponential stability of impulsive stochastic functional differential systems. Stat. Probab. Lett. 80, 1854-1862 (2010)

28. Di, C, Jiang, D, Shi, N: Multigroup SIR epidemic model with stochastic perturbation. Physica A 390, 1747-1762 (2011)

29. Konishi, K: Time-delay-induced stabilization of coupled discrete-time systems. Phys. Rev. E 67, 017201 (2013)

30. Chen, H, Sun, J: Stability analysis for coupled systems with time delay on networks. Physica A 391, 528-534 (2012)

31. Hale, JK: Ordinary Differential Equations. Dover, New York (2009)

32. Mao, X, Yuan, C: Stochastic Differential Equations with Markovian Switching. Imperial College Press, London (2006)

33. Chen, F, Huang, A: On a nonautonomous predator-prey model with prey dispersal. Appl. Math. Comput. 184, 809-822 (2007) 